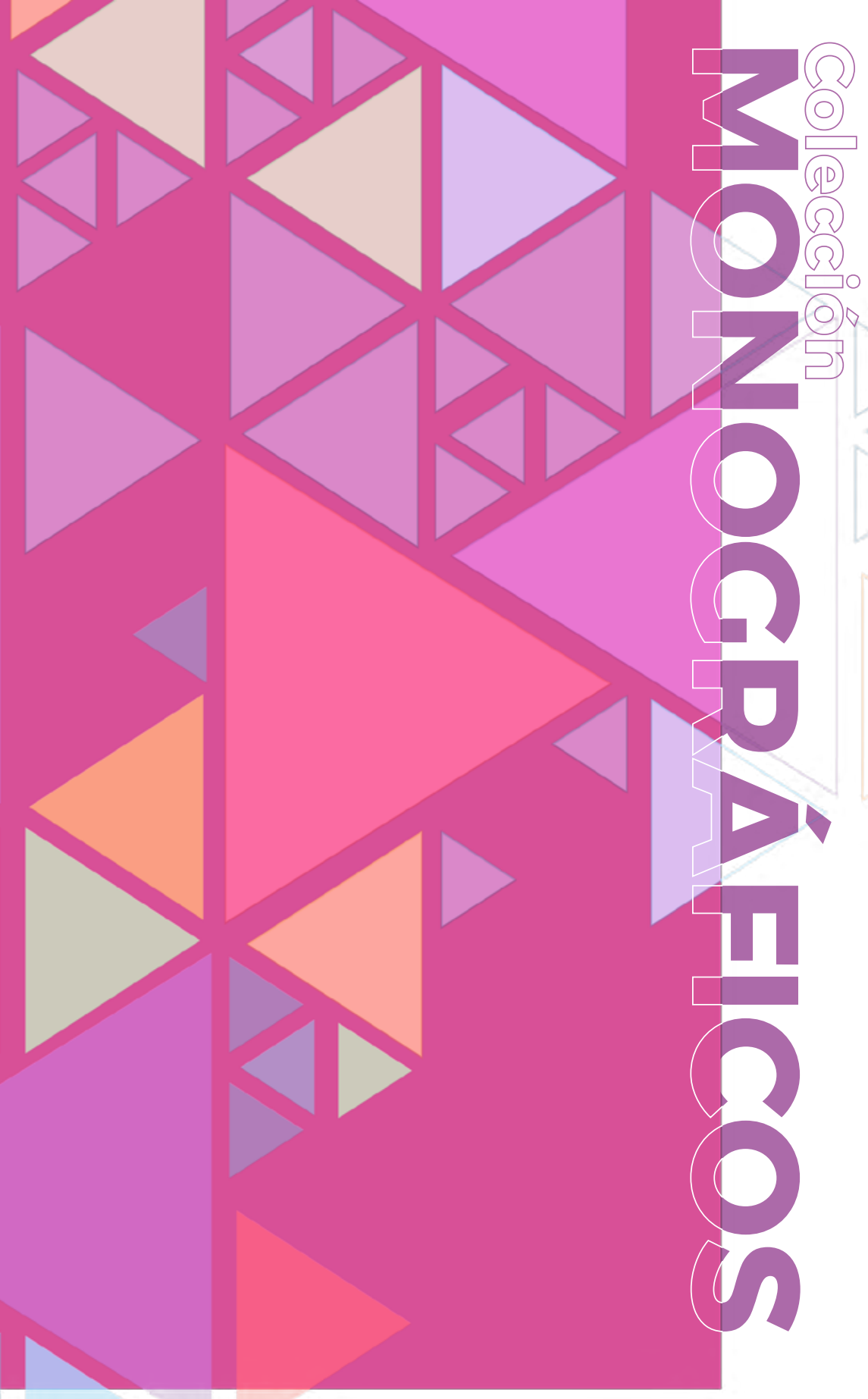

Responsabilidad social empresarial:

Prácticas y perspectivas de la gestión organizacional en el contexto ecuatoriano

Mariana Del Rocío Verdezoto Reinoso Compiladora

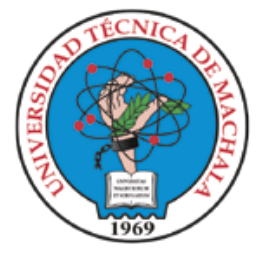


Ediciones UTMACH

147 pág: $21 \times 29,7 \mathrm{~cm}$

Colección Monográficos

Título: Responsabilidad social empresarial: prácticas y perspectivas de la gestión organizacional en el contexto ecuatoriano - Mariana

Del Rocío Verdezoto Reinoso (Compiladora)

Primera edición 2020

ISBN: 978-9942-24-140-5

CDD 338,9

1. Desarrollo y crecimeinto económico,

2. Empresa y gestión

--Temas relacionados

Publicación PDF 
Responsabilidad social empresarial: prácticas y perspectivas de la gestión organizacional en el contexto ecuatoriano

Mariana Del Rocío Verdezoto Reinoso

COMPILADORA

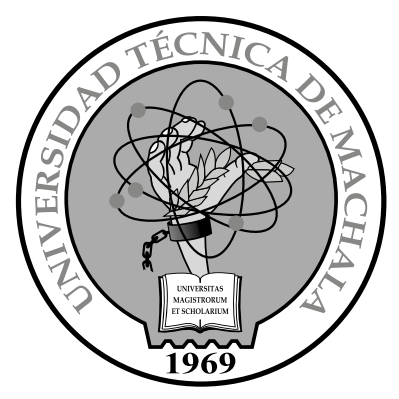


Autoridades

César Quezada Abad - Rector Amarilis Borja Herrera - Vicerrector Académico Jhonny Pérez Rodríguez - Vicerrector Administrativo

Luis Brito Gaona

Director de Investigación

(c) Ediciones UTMACH Colección Monográficos

Título original:

Responsabilidad social empresarial: prácticas y perspectivas de la gestión organizacional en el contexto ecuatoriano

ISBN: 978-9942-24-140-5

(c) Mariana Del Rocío Verdezoto Reinoso

(Compiladora)

(c) Autores de capítulos

DOI: http://doi.org/10.48190/9789942241405

Primera edición 2020

Karina Lozano Zambrano Jefe editor / Diseño y edición editorial

Fernanda Tusa Jumbo - Corrector de estilos Jorge Maza-Cordova - Asesor tecnológico

Karla Ibañez y Cyndi Aguilar - Equipo de difusión

Este obra está bajo una licencia de Creative Commons Reconocimiento No Comercial.

Esta licencia permite a otros entremezclar, ajustar y construir a partir de su obra con fines no comerciales, siempre y cuando le reconozcan la autoría y sus nuevas creaciones estén bajo una licencia con los mismos términos. 


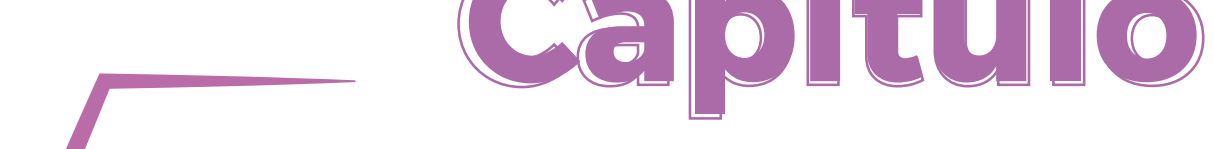

Responsabilidad social en el sector público del Ecuador

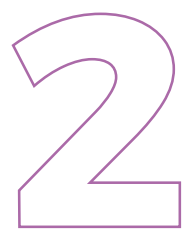


Responsabilidad social en el sector público del Ecuador

\author{
Zaida Patricia Morocho Román \\ Eduardo Vinicio Pulla Carrión \\ Ernesto Xavier González Ramón
}

Autores 


\section{Responsabilidad social en el sector público del Ecuador}

\section{Zaida Patricia Morocho Román}

Ingeniera Comercial, Magister en Tributación por la Escuela Superior Politécnica del Litoral, Certificación Internacional en NIIF por el Instituto Tecnológico de Monterrey-México, Doctorando de la Universidad del Zulia-Venezuela. Con experiencia profesional en el sector público y privado. Actualmente Docente Titular de la Universidad Técnica de Machala.

\section{Eduardo Vinicio Pulla Carrión}

Catedrático Universitario desde el año 2008, actualmente Docente Titular de la Universidad Técnica de Machala, Ingeniero Comercial, Magister en Administración de Empresas por la Universidad Nacional de Loja. Actualmente continúa sus estudios de Doctorado en la Universidad de Zulia, Venezuela.

\section{Ernesto Xavier González Ramón}

Abogado de los Tribunales y Juzgados de la República del Ecuador - Magister en Derechos Fundamentales y Justicia Constitucional - Profesor Titular de la Universidad Técnica de Machala - Autor de Artículos relacionados a Educación y Derecho.

DOI: http://doi.org/10.48190/9789942241405.2 


\section{Resumen}

A partir del análisis de bases bibliográficas respecto de responsabilidad social, normas y acuerdos sobre el interés en la implementación de compromisos prospectivos para alcanzar el desarrollo sostenible, la presente investigación realiza una aproximación desde la perspectiva de la gestión pública y su grado de corresponsabilidad ética en las prácticas de buen gobierno mediante el análisis de las prácticas de responsabilidad social aplicadas en el sector público, frente a sus principales categorías, derivadas de la teoría expuesta, para orientar los cambios estructurales en la redefinición de políticas públicas incluyentes que se alineen a los objetivos de desarrollo según el Plan Nacional del Desarrollo 2017-2021 -Toda una Vida-, este alcance inicial se realiza mediante un diagnóstico y evaluación de la situación actual de las prácticas de responsabilidad social adoptadas en el sector público en el Ecuador, en busca de un equilibrio en la gestión en contextos similares basados en los efectos de buenas prácticas de responsabilidad en el sector público. Dentro de los instrumentos considerados importantes, está el marco normativo y las leyes que regulan la gestión pública, ya que señala el camino que permite ampliar el ámbito de acción y medir su ejecución, estableciendo las debilidades y fortalezas institucionales que posteriormente son los insumos que generan un plan de acción para mejorar la gestión de la responsabilidad social de las instituciones públicas, lo que contribuye al progreso de la sociedad mediante los valores de transparencia, ética política, participación ciudadana e igualdad, generando más confianza entre el Estado y la Sociedad.

Palabras claves: responsabilidad social, sector público, gestión.

\section{Introducción}

Las manifestaciones asociadas a la Responsabilidad Social en el sector público generan incentivos de buenas prácticas que permite tensionar tanto la filosofía como la gestión organizacional, generando los círculos de mejora continua y de sostenibilidad en la forma de hacer las cosas.

La integración voluntaria de las preocupaciones sociales y de las exigencias de los ciudadanos en las decisiones de los poderes públicos implica una aproximación al modelo socialmente responsable; como también al ajuste de la política a las peticiones ciudadanas y un uso responsable de la misma. No obstante, el voluntarismo inherente a la Responsabilidad Social y su carácter para jurídico comienza a ceder en su traslación a la esfera pública, a través de su mutación en normativa legal y exigible. (Ruiz \& Ruiz, 2014, pág. 4) 
El Estado y, por lo tanto, todas las instituciones públicas, por definición, deben ejercer un rol en lo social, cuyo objetivo prioritario es la generación de políticas públicas en beneficio de toda la ciudadanía. Bajo este contexto, se podría decir que, la Responsabilidad Social es parte inherente de la política del Estado.

González y León (2013), sostienen que las administraciones públicas han concientizado la importancia de la gestión de responsabilidad social, poniendo en marcha políticas orientadas a la administración integrada como estrategia a largo plazo, que se traduce en beneficios económicos y sociales. En este sentido, las instituciones públicas transforman el contexto de la toma de decisiones empresariales.

Los poderes públicos tienen distintos tipos de responsabilidad según el ámbito y las obligaciones por las que tienen que responder, por lo que se puede decir que la responsabilidad política o responsabilidad administrativa de los poderes públicos se deriva de la obligación de actuar como garantes de los derechos de los ciudadanos y del interés general. Por lo que responsabilidad social pública implica un deber más, que es el de vincularse en la defensa del interés general mediante la protección y promoción de los bienes y derechos que ésta propone, garantizando las expectativas de la ciudadanía (Puig i Campmany \& Martínez i Hernández, 2008).

La Comisión de las Comunidades Europeas (CCE, 2001) define a la Responsabilidad Social Empresarial, como el instrumento mediante el cual las empresas integran aspectos sociales y ambientales en sus operaciones y en la interacción con sus grupos de interés, donde más allá de las exigencias legales lo hacen con interés y voluntad. Por lo que Responsabilidad Social se entiende como una contribución empresarial al desarrollo sostenible con enfoque de gestión que impulsa la competitividad y cohesión social.

Para la Comisión Económica para América Latina (CEPAL, 2004), la Responsabilidad Social es la forma de hacer negocios considerando los efectos sociales, ambientales y económicos de la acción empresarial integrando en ella el respeto por los valores éticos, las personas, las comunidades y el medio ambiente.

Desde la mirada de (Viteri \& Jácome, 2011, págs. 3-9), es el compromiso de la empresa para identificar los problemas que afecten a sus grupos de interés y emprender acciones que generen impactos sociales, económicos y ambientales positivos, con el aporte de soluciones basadas en la transparencia, pluralidad, sustentabilidad y ética; la meta es el desarrollo sustentable del ser humano y de su entorno.

En este contexto, la responsabilidad social es un compromiso de las organizaciones públicas y privadas para contribuir al desarrollo sostenible, trabajando con los empleados y sus familias, con la comunidad y la sociedad para mejorar la calidad de vida, cuyo objetivo es que tanto las organizaciones como la comunidad obtengan beneficios mutuos (Canovas García \& Mateos García, 2014).

El concepto de Responsabilidad Social Corporativa (RSC) está intrínsecamente ligado al desarrollo sostenible definido en el Informe Bruntdland de la Organización 
de las Naciones Unidas ONU (2015) que condiciona de forma permanente, tanto a agentes privados como a públicos, en la medida en que sus actividades deben "satisfacer las necesidades del presente sin poner en peligro la capacidad de las generaciones futuras para satisfacer las suyas propias” (p.1).

El desarrollo sostenible es un tema en el cual participan actores tanto a nivel nacional como internacional. Las reformas económicas, sociales y estructurales que requieren muchos países necesitan de un gobierno eficiente, responsable, transparente y con mayor participación ciudadana, que permita una mejor y más rápida respuesta a los retos de democratización y rendición de cuentas, de la globalización económica, la descentralización del poder a niveles locales, y la corrección de desequilibrios sociales (Banco Mundial, 2000).

La tradicional voluntariedad en la ejecución de las acciones de responsabilidad social corporativa, se debe complementar con una actuación por parte del Estado materializada en la implementación de políticas públicas que permitan promover, incentivar y consolidar prácticas responsables (Lozano, Albareda, Ysa, Rosher, \& Marcuccio, 2005).

Las políticas públicas se encuentran relacionadas de forma directa con la responsabilidad social ya que es la manera que tiene el estado para dar respuesta o soluciones a las demandas de la sociedad, con la finalidad de promover mejores estándares sociales, económicos y medio ambientales. Fox, Ward, \& Howard (2002) afirma:

Las políticas públicas en materia de responsabilidad social corporativa se clasifican en:

- Políticas reguladoras del sector público, mediante la cual se definen los estándares mínimos de la actuación empresarial dentro del marco legal.

- Políticas facilitadoras o de apoyo, que permiten estimular la implicación de actores clave a través de la puesta en marcha de formación, comunicación, o de difusión.

- Políticas de colaboración y acuerdos estratégicos, pueden agrupar y poner en común las capacidades y los logros de los sectores públicos y privado y la sociedad civil.

- Políticas de promoción o reconocimiento directo de los esfuerzos de otras organizaciones, o el mismo efecto ejemplarizante que tienen las prácticas de gestión del sector público. (p.6)

El impacto de éstas políticas no sólo se entiende en la relación tradicional de la acción de los gobiernos hacia el sector privado, sino que permite la existencia de una relación multilateral y puede orientarse a su integración en la propia administración pública, en la relación entre gobierno y sociedad civil; o en a una relación multilateral entre gobiernos, empresa y sociedad civil (Lozano et al., 2005).

La condición general es que las organizaciones ejercen su responsabilidad social cuando su visión enmarca las expectativas que tienen sus diferentes grupos 
de interés (stakeholders), acerca de sus comportamientos. En el contexto de una nueva gestión pública, la intersectorialidad entendida como el trabajo coordinado con otro tipo de organizaciones (empresas, organizaciones sociales y ciudadanas) y otras instituciones estatales, promueve la conformación de redes que optimizan el uso de recursos y enfoques, dirigiéndoles en forma eficiente hacia la satisfacción de las necesidades de la sociedad. Al mismo tiempo, los cambios en las demandas y aspiraciones de la sociedad civil, hacen necesario incorporar la "transversalidad" en la implementación de la política pública, a partir de la generación de buenas prácticas, en un modelo de responsabilidad social que se caracterice por atender estas nuevas demandas como variables dinámicas en los procesos de gestión pública (Zenck Huerta, Rios Rivera, Pogo García, \& Cueto Cedillo, 2017).

En la actualidad, el modelo de gestión para la responsabilidad social ha cambiado. Se pasa de un modelo donde los ciudadanos son clientes y consumidores, a otro donde son socios, capaces de participar (Navarro Galera, Ruiz Lozano, De Los Rios Berjillos, \& Tirado Valencia, 2013). Analizando esta teoría, los autores indican que la responsabilidad social de las organizaciones no debería estar basada en logros, sino en relación con las demandas de los stakeholders, esto con el propósito de dirigir esfuerzos de una manera más organizada, eficiente y oportuna, convirtiéndolos en participantes de un cambio. También se presenta una gran modificación al interior de la organización, teniéndose que implementar políticas de responsabilidad social en los procesos de planificación, considerando el punto de vista de los grupos de interés involucrados.

Hoy en día surgen nuevos elementos que enriquecen a la responsabilidad social empresarial, por ejemplo, la moral y la ética; la problemática ambiental y la sostenibilidad aparecen en el debate conceptual y entran a formar parte concomitante de la definición de la responsabilidad social empresarial, para ampliarla y complementarla, dándole mayor trascendencia dentro de los modelos de gestión empresarial (Orjuela Cordova, 2011).

Las entidades del sector público realizan su gestión hacia la ciudadanía más allá de la racionalidad económica, por tal razón la información sobre sostenibilidad en el ámbito público toma un papel fundamental para la evaluación de una gestión transparente y responsable (Navarro et al., 2013). Es importante que este sector genere información que reporte riesgos financieros futuros, oportunidades y debilidades de su gestión, buscando definir aspectos claves para la sostenibilidad.

Las políticas públicas de responsabilidad social corporativa, encuentran su antecedente en el marco normativo internacional con distintas convenciones, declaraciones y guías (CCE, 2001).

1. Pacto Global de Naciones Unidas (United Nations Global Compact).

2. Principios Rectores de las Naciones Unidas sobre Empresas y Derechos $\mathrm{Hu}-$ manos (Observatorio de Responsabilidad Social Corporativa, 2003).

3. ISO 26000 (International Organization for Standardization). 
4. Declaración tripartita de principios sobre las empresas multinacionales y política social Organización Internacional del Trabajo (OIT, 2001).

5. Directrices para Empresas Multinacionales. Organización para la Cooperación y el Desarrollo Económico (OCDE, 2011).

En el año 2015, la ONU aprobó la Agenda 2030 sobre el Desarrollo Sostenible, una nueva oportunidad para que los países emprendan un camino para mejorar la vida de todos. La agenda cuenta con 17 Objetivos de Desarrollo Sostenible (ODS), los que incluyen erradicar la pobreza, proteger el planeta, la educación, la prosperidad para todos como parte de una nueva agenda de desarrollo sostenible. Cada objetivo tiene metas específicas que deben alcanzarse en los próximo 15 años; para alcanzarlas debe existir una interrelación entre los gobiernos, el sector privado y la sociedad civil (Organización de las Naciones Unidas).

Mediante la propuesta de los ODS, se busca dar continuidad al compromiso adquirido por los países, para abordar problemas de acción urgente que representan desafíos para todos; gobierno, organizaciones, sociedad civil; este contexto se vincula de forma directa con la Responsabilidad Social especialmente en los ODS 16 y 17; promover sociedades justas, pacíficas e inclusivas y revitalizar la alianza mundial para el desarrollo sostenible, respectivamente.

Internacionalmente se han desarrollado estándares que ayudan a las organizaciones a revelar la ejecución de sus planes de acción en desarrollo sostenible y responsabilidad social. El Global Inititiative Reporting GRI-G4 (GRI Empowering Sustainable Decisions) son los primeros estándares globales para informes de sostenibilidad; cuentan con una estructura modular, interrelacionada, y representan la mejor práctica global para informar sobre una gama de impactos económicos, ambientales y sociales, otras de las asociaciones profesionales que han contribuido a normalizar y transparentar la información sobre responsabilidad social, por ejemplo en Ecuador el Consorcio Ecuatoriano para la Responsabilidad Social (CERES) y en España, la Asociación Española de Contabilidad (AECA).

En el Ecuador, el Estado tiene como prioridad, prestar servicios a la comunidad, cumpliendo con los principios de eficacia, eficiencia y transparencia conforme lo establece la Constitución de la República del Ecuador (2008), la acción de estas actividades, los planes y programas en ejecución, metas e informes de gestión e indicadores de desempeño, se transparenta con la difusión de la información pública a través del portal de información o página web mediante el mecanismo de rendición de cuentas a la ciudadanía, de acuerdo a lo que establece el Art. 7 de la Ley Orgánica de Transparencia y Acceso a la Información Pública LOTAIP (Asamblea Nacional, 2004).

Todas las instituciones públicas en Ecuador se encuentran reguladas a través de un marco legal y normativo, el mismo que contiene procesos, modelos de gestión, indicadores, seguimiento y evaluación de resultados; en fin, todo un esquema pro- 
tocolario a cumplir, que si bien es cierto se cumple o no, no es el tema de discusión, sino el cómo se cumple y cuál es la repercusión por la forma de ejecutar dichos procesos.

No se puede dejar de mencionar la gran ventaja que tienen las instituciones del sector público frente a otras organizaciones cuyo fin es totalmente diferente, dada su naturaleza. Sin embargo, el sector público tiene un "deber" respecto a la implementación coordinada y planificada de buenas prácticas en materia de responsabilidad social, a pesar de haber impulsado la implementación de una serie de herramientas de gestión, por mencionar un ejemplo, en el año 2010 entra en vigencia el proyecto Gobierno por Resultados (GPR), herramienta que presenta algunos objetivos como el de mejorar la ejecución del presupuesto gubernamental, mejorar la calidad de toma de decisiones, orientar al gobierno en las áreas de mayor urgencia de mejora, estimular la colaboración entre las instituciones del Estado, ayudar en el desmantelamiento de los procesos burocráticos, desarrollar competencias institucionales en la planificación estratégica, operativa y de gestión de resultados; entre otras, es decir un proyecto que enmarca casi todos los enfoques de responsabilidad social tanto para los actores internos como externos (Norma Técnica de Implementación y Operación de la metodología y herramienta de Gobierno por Resultados, 2011).

\section{Justificación}

Hay que mencionar, que en la gran mayoría de instituciones públicas existe una cultura establecida para hacer las cosas, que limita la implementación de nuevas ideas porque "siempre se han hecho las cosas así", esto conlleva a mantener la gerencia tradicional y no dar paso a nuevos retos que coadyuven a mejorar la gestión pública.

Para vencer estas dificultades, es necesario que el Estado, sus instituciones y sus directivos, revisen sus estructuras, competencias, funciones, procesos, directrices, etc. y puedan definir las mejores estrategias para que los resultados sean mejores.

Se requiere conocer, también cómo la responsabilidad social puede ser aplicada a nivel de instituciones públicas, debido a que el enfoque ha sido la aplicación de acciones a nivel empresarial privado.

Por todo lo expuesto, la manifestación de responsabilidad social en el sector público, promueve en las instituciones públicas la revisión de sus objetivos y metas institucionales, su desempeño considerando el concepto "socialmente responsable" (social, económico, de medio ambiente y su impacto en los stakeholders, preocupándose de mejorar continuamente su gestión para satisfacer a la ciudadanía (cliente principal del Estado), lo que contribuye directamente con el desarrollo del país.

La investigación de este tema busca la inserción de medidas adecuadas en la toma de conciencia, de no solo pensar en sí mismo sino en el entorno institucional a beneficio de la ciudadanía, lo que conlleva a plantear una alternativa de trabajo 
que permita mejorar las fortalezas y eliminar las debilidades mediante un plan de acción ejecutable y flexible donde se evidencie el alcance de metas institucionales con el fin de cumplir lo que abarca el ámbito de responsabilidad social institucional.

A partir de la conceptualización teórica de desarrollo sostenible y responsabilidad social, y en observancia a la política social establecida en el país, desde el Plan Nacional de Desarrollo (PND) 2017-2021, el cual enmarca la búsqueda de la transparencia de la gestión pública y privada, el Estado en todo su contexto, ha impulsado leyes y procesos que fomentan la transparencia, mediante herramientas que promueven la participación ciudadana, el acceso a la información y la rendición de cuentas; por lo que de acuerdo al enfoque del eje 3 "Más sociedad, mejor Estado" desarrollado en el Plan Nacional de Desarrollo, busca la transparencia de la gestión pública y privada, por tanto, esta investigación se vincula de forma directa con el Objetivo 8 del PND donde se busca "Promover la transparencia y la corresponsabilidad para una nueva ética social”, desprendiéndose de éste, el objetivo fomentar prácticas socialmente responsables en la gestión pública, como aporte significativo para el funcionamiento general y mejorar los procesos en la institución, generando confianza y valor agregado en la implementación de acciones a beneficio de la ciudadanía (Senplades, 2017).

Así mismo, se considera que el control interno es un proceso integral orientado a cumplir con el ordenamiento jurídico, técnico y administrativo, promoviendo la eficacia y la eficiencia de las operaciones de la entidad y garantizar la confiabilidad y oportunidad de la información, así como la adopción de medidas oportunas para corregir las deficiencias de control (Contraloría General del Estado, 2009).

El impacto y repercusión del no cumplimiento o cumplimiento a medias del marco normativo, ante los hechos y circunstancias originadas en las instituciones del sector público, recae de manera directa sobre la ejecución del presupuesto institucional, derivándose otros aspectos negativos, como el mal uso e inversión de los recursos económicos, no optimización del presupuesto, bienes de larga duración sin ser utilizados, bienes adquiridos por necesidad personal y no institucional; si bien es cierto, en un primer momento el tema de responsabilidad social quería tornarse como una obligación gubernamental pero con el pasar del tiempo se establece que este tema fuese una adopción voluntaria por parte de las entidades tanto del sector público como privado, considerando aquello se determina, que es una herramienta de gestión más que de carácter obligatorio, necesaria, dado que el impacto en las entidades públicas aborda el contexto social, económico y de medio ambiente.

El presente trabajo investigativo, se aplica en las instituciones del sector público no financiero, con el objetivo de mejorar sus procesos, los mismos que necesitan generar confianza, tendientes a lograr la recuperación de espacios públicos, para el disfrute de la comunidad. 


\section{Objetivo general}

Analizar las prácticas de responsabilidad social aplicadas el sector público, frente a sus principales categorías.

\section{Objetivos específicos}

1. Diagnosticar las principales manifestaciones de responsabilidad social adoptadas en el sector público.

2. Evaluar las prácticas de responsabilidad social en el sector público en el Ecuador.

\section{Metodología}

Se trata de un trabajo de investigación de carácter exploratorio, donde se emplean métodos cualitativos acordes a un enfoque inductivo.

La metodología utilizada, inicia con la investigación de bibliografía y las principales referencias internacionales en materia de gestión socialmente responsable. Con esta aplicación se busca determinar el marco de aplicación existente, así como la revisión crítica de la literatura de campo. Con ello se identifica las fuentes principales para la determinación del marco conceptual del trabajo, señalando definiciones y elementos que lo conforman.

De acuerdo a Hernández, Fernández \& Baptista (2006), las investigaciones cualitativas se fundamentan en un proceso inductivo (explorar y describir, y luego generar perspectivas teóricas). Van de lo particular a lo general. Por ejemplo, en un estudio cualitativo, el investigador entrevista a una persona, analiza los datos que obtuvo y saca algunas conclusiones; posteriormente, entrevista a otra persona, analiza esta nueva información y revisa sus resultados y conclusiones; del mismo modo, efectúa y analiza las entrevistas para comprender lo que busca. Es decir, procede caso por caso, dato por dato, hasta llegar a una perspectiva más general.

Así mismo, se revisa la normativa específica existente. En primera instancia la emitida por los organismos internacionales, a continuación, las políticas públicas que rigen en el Ecuador, así como las normas que rigen la responsabilidad social. Finalmente se revisan informes de organismos públicos y guías de buenas prácticas o de buen gobierno, especialmente del sector público. 


\section{Materiales y métodos}

En el proceso investigativo se pretende analizar las prácticas de responsabilidad social aplicadas en el sector público, iniciando por el diagnóstico y posterior evaluación de las manifestaciones aplicada; bajo este esquema, como parte del diseño metodológico de investigación y con la finalidad de definir las prácticas de responsabilidad social, se realiza una investigación por el método exploratorio y descriptivo, con un enfoque cualitativo; con el afán de describir, analizar y evaluar los resultados encontrados.

Para realizar el análisis de la información cualitativa se estableció parámetros comparativos y de ejecución de herramientas a aplicar en la responsabilidad social.

\section{Resultados y Discusión}

Al ser un estudio exploratorio se precisa una herramienta adaptable que incluya elementos cuya pertinencia ha sido contrapuesta con la normativa, competencias legales y la consideración de los ODS para su adecuada aplicación. La herramienta - Listado de Comprobación - original contiene 112 ítems y está dividida en cuatro secciones principales.

Tabla 1. Listado de Comprobación

\begin{tabular}{|l|c|}
\hline \multicolumn{1}{|c|}{ Secciones } & Ítems \\
\hline Información sobre el desempeño general de la entidad & 39 \\
\hline $\begin{array}{l}\text { Información sobre el desempeño de responsabilidad } \\
\text { social a nivel económico }\end{array}$ & 27 \\
\hline $\begin{array}{l}\text { Información sobre el desempeño de responsabilidad } \\
\text { social a nivel social }\end{array}$ & 24 \\
\hline $\begin{array}{l}\text { Información sobre el desempeño de responsabilidad } \\
\text { social a nivel ambiental }\end{array}$ & 22 \\
\hline
\end{tabular}

Fuente: Adaptado de (Navarro Galera, Alcaraz Quilez, \& Ortiz, 2010)

Bajo este esquema se busca la posibilidad de realizar un análisis basado en la teoría expuesta sobre las principales categorías de la responsabilidad social.

El listado de comprobación adaptado al contexto local, luego de la revisión de la normativa, competencias legales y la consideración de los ODS vinculados al modelo de gestión del Ministerio del Interior, permitiría evaluar la información económica, social y medioambiental. 
Tabla 2. Componentes del análisis de información

\begin{tabular}{|l|}
\hline BLOQUE I. Información sobre el desempeño general del Ministerio del Interior \\
\hline Enfoque político-estratégico de la RS (Responsabilidad Social) \\
\hline Enfoque ético-buen gobierno y transparencia \\
\hline Enfoque integrador de los Grupos de Interés \\
\hline BLOQUE II. Información sobre el desempeño de la RS a nivel económico \\
\hline Información económica, contable y presupuestaria \\
\hline Indicadores de ingresos y gastos \\
\hline Indicadores de Control Interno \\
\hline BLOQUE III. Información sobre el desempeño de la RS a nivel social \\
\hline Responsabilidad en la gestión del personal del Ministerio del Interior \\
\hline Relaciones y operaciones con proveedores y contratistas \\
\hline Relaciones y operaciones con la comunidad \\
\hline BLOQUE IV. Información sobre el desempeño de la RS a nivel ambiental \\
\hline Información sobre políticas medioambientales \\
\hline
\end{tabular}

Nota: Adaptado de (Navarro Galera, Alcaraz Quilez, \& Ortiz, 2010)

El análisis realizado mediante los instrumentos de revisión bibliográfica y documental, se complementa con la observación y hallazgos; además de asegurar el acceso a la información de la ciudadanía de acuerdo a lo que establece la ley, se hace necesario verificar la calidad, pertinencia y veracidad de dicha información, con el fin de concretar políticas públicas para una gestión responsable con desarrollo sostenible (Zenck Huerta, Rios Rivera, Pogo García, \& Cueto Cedillo, 2017). Dicho modelo de gestión debe contribuir a la consolidación de una política de responsabilidad social corporativa y de donde se deriven insumos para una eficiente transparencia del desempeño del Ministerio del Interior del Ecuador y las necesidades de información de sus grupos de interés.

La coherencia entre la práctica de estrategias de responsabilidad social y la información oportuna, se debe demostrar en el cumplimiento de los indicadores que conforman las dimensiones de los componentes de la herramienta planteada para el diagnóstico de acuerdo al cuadro No. 2.

De acuerdo al principio de transparencia de responsabilidad social presente en la ISO 26000, las organizaciones deben suministrar toda la información requerida por las partes interesadas, de esta forma, pueden tomar decisiones basadas en datos presentados de forma completa, comprensible, receptiva, precisa, equilibrada, oportuna y accesible (Argandoña \& Isea Silva, 2011). Como el acceso a la información pública es una garantía constitucional regulada por la Ley Orgánica de Transparencia y Acceso a la Información (LOTAIP 2004), los grupos de interés no sólo deben estar informados por parte del Ministerio del Interior sobre los programas 
que promueven el desarrollo sostenible, sino que además el marco legal ecuatoriano les garantiza el acceso de manera abierta a cierta información de la gestión administrativa de la entidad, por pertenecer al sector público (Zenck et al., 2017).

Como punto de partida para dimensionar los resultados del estudio, se presenta un mapa de la construcción de las prácticas de responsabilidad social que ejecuta el Ministerio del Interior; luego, una breve descripción de los programas que generan las interacciones y relaciones con sus grupos de interés; y finalmente, su compromiso con la transparencia de la información que brinda en sus páginas web oficiales, a través del Listado de Comprobación, de tal forma que se pueda validar las políticas de responsabilidad social ejecutadas y las necesidades de información que tienen los grupos de interés y ciudadanía en general.

Siendo el Ministerio del Interior una institución pública, cuyo objetivo institucional es garantizar la convivencia social pacífica y la seguridad ciudadana de todos los ecuatorianos, teniendo como visión que la seguridad ciudadana y convivencia posesiona al ser humano como el centro de las políticas, programas y proyectos para garantizar el derecho de las personas a ser y sentirse protegidos de la violencia y cometimiento de infracciones (delitos y contravenciones); teniendo como ejes estratégicos, la previsión, prevención y respuesta, el ministerio ha venido desarrollando durante mucho tiempo programas de ayuda y apoyo a la comunidad independientemente de la labor constitucional encomendada como parte de prácticas de responsabilidad social.

Tabla 3. Acciones de Responsabilidad Social del Ministerio del Interior

\begin{tabular}{|c|c|}
\hline Denominación/Proyecto & Acción/ Grupo beneficiado \\
\hline Hipoterapia & $\begin{array}{l}\text { Dirigido a personas con capacidades especiales, motivando e incentivando a mejorar sus } \\
\text { destrezas; lo realizan con la Unidad de Equitación y Remonta UER. }\end{array}$ \\
\hline Caravana de la Salud & $\begin{array}{l}\text { Acción de RS donde a través de sus profesionales de higiene y salud, brindan atención y } \\
\text { asistencia a poblaciones marginales. }\end{array}$ \\
\hline Pirotecnia responsable & $\begin{array}{l}\text { Difunde a la ciudadanía, las causas y efectos producidos por el mal uso de material piro- } \\
\text { técnico. }\end{array}$ \\
\hline Yo educo a mi mascota & $\begin{array}{l}\text { Capacita a la ciudadanía en adiestramiento canino con ejercicios prácticos, para que ten- } \\
\text { gan el control de sus mascotas y puedan corregir ciertas conductas no deseadas. }\end{array}$ \\
\hline Alas de inclusión & $\begin{array}{l}\text { Se coordina la participación de instituciones que trabajan con niños de capacidades dife- } \\
\text { rentes, a fin de programar sobrevuelos con el helicóptero de la institución e incentivar la } \\
\text { recuperación y realización de sueños anhelados. }\end{array}$ \\
\hline $\begin{array}{l}\text { No arranques la piel de } \\
\text { los bosques }\end{array}$ & $\begin{array}{l}\text { Charlas en planteles educativos, con el fin de dar a conocer la importancia de la conser- } \\
\text { vación de los ecosistemas. }\end{array}$ \\
\hline $\begin{array}{l}\text { Grupo lúdico Paquito y } \\
\text { sus amigos }\end{array}$ & $\begin{array}{l}\text { A través del personal capacitado en artes lúdicas, se realizan estrategias de prevención para } \\
\text { fortalecer la formación en temas de prevención, seguridad ciudadana, principios y valores } \\
\text { a niños, niñas y adolescentes. }\end{array}$ \\
\hline
\end{tabular}




\begin{tabular}{|l|l|}
\hline Grupo Teatrino & $\begin{array}{l}\text { Es una acción de RS que va en beneficio de los niños, niñas y adolescentes. Consiste en } \\
\text { capacitar a niños y niñas comprendidos entre las edades de 5 a } 10 \text { años, sobre temas de } \\
\text { autoprotección, seguridad, bullying, derechos y obligaciones. }\end{array}$ \\
\hline $\begin{array}{l}\text { Feria de Seguridad Ciu- } \\
\text { dadana }\end{array}$ & $\begin{array}{l}\text { Acción que realiza la institución a través de sus diferentes servicios para estrechar lazos } \\
\text { con la sociedad y que ésta conozca las diferentes actividades extras de la institución, en } \\
\text { donde se fomentan temas como: charlas de solidaridad y convivencia pacífica ciudadana, } \\
\text { recuperación de valores y principios cívicos, etc. }\end{array}$ \\
\hline Campaña de Prevención & $\begin{array}{l}\text { Programa que realiza la entidad, a través de los servidores policiales en cada uno de los } \\
\text { circuitos en la cual se analiza y se realizan estrategias en los temas de mayor incidencia } \\
\text { delictual, peligrosa y violenta. }\end{array}$ \\
\hline
\end{tabular}

Si bien la responsabilidad social es un compromiso asumido de forma voluntaria y que va más allá de lo que exige la ley, para el Ministerio del Interior, la responsabilidad social se convierte en una voluntad política a ser promovida desde las prácticas socialmente responsables, tanto dentro de la institución como fuera de ella y esta se ve reflejada en el desarrollo de sus estrategias y programas que marcan el liderazgo, tal como lo demuestra el haber alcanzado el reconocimiento Ecuatoriano Ambiental Punto Verde ${ }^{1}$, que lo logró a través de la evaluación comparativa de indicadores en la medición de las actividades en los ejes temáticos como: la gestión de deshechos, gestión de papel, uso eficiente de agua, energía y combustibles; capacitación y compras responsables.

El bloque político, incorpora el enfoque sobre la estrategia de responsabilidad social, además del buen gobierno, la transparencia y la relación con los grupos de interés. La mayoría de los ítems se encuentran publicados en las diferentes páginas web del Ministerio del Interior; la normativa base para una estrategia de responsabilidad social está al acceso de la ciudadanía, como la planificación estratégica, los objetivos, misión, visión, servicios ofrecidos, los canales de participación abiertos a los usuarios para el entendimiento de las prácticas de responsabilidad social, consultas, opiniones, sugerencias o denuncias que podrán hacer los ciudadanos; siendo sumamente importantes en relación a la rendición de cuentas.

El bloque económico es el que cuenta con mayor número de ítems publicados. $\mathrm{Al}$ analizar la totalidad de la información publicada, se constata que es bastante completa. Indicadores macroeconómicos, presupuestos, informes sobre transferencias, liquidez, superávit, ingresos fiscales e inversión.

El bloque de desempeño social tiene un alto porcentaje de publicación en lo referente a las relaciones y operaciones con la comunidad, ya que los ítems se enfocan en el servicio a la sociedad, que es un aspecto central al momento de hablar de responsabilidad social. Se puede notar en cambio que la información publicada

${ }^{1}$ El Ministerio del Ambiente del Ecuador, con el objetivo de incentivar al sector público y privado, a emplear nuevas y mejores prácticas productivas y de servicios 
relacionada a proveedores y contratitas es escasa y muy general; y en cuanto a sus servidores públicos, la información es escasa, dejando fuera información básica como salarios, rotación, antigüedad y formación. La no publicación de estos aspectos podría denotar que se considera un aspecto importante en la rendición de cuentas.

En el bloque medioambiental, se publica la implementación de la política ambiental, capacitación continua y campañas de comunicación interna que se ejecutan. Éstas le permitieron alcanzar el reconocimiento ecuatoriano ambiental Punto Verde.

\section{Conclusiones}

Durante el desarrollo de este trabajo de investigación, se evaluó las prácticas de responsabilidad social en el sector público en el Ecuador, tomando como objeto de estudio al Ministerio del Interior, entidad que realiza acciones sociales encaminadas a desarrollar plenamente la RS. En la actualidad dichas acciones son llevadas a cabo desde el escenario de la administración pública como un factor de desarrollo para la ciudadanía en general, impulsando así la participación de manera activa e incluyente.

El fortalecimiento del modelo de gestión del Ministerio del Interior se promueve mediante el desarrollo ciudadano; a través de la aplicación de una estrategia desde la organización y participación ciudadana para intervenir en su entorno y mejorar sus condiciones de vida, actuando de manera comprometida, consciente y crítica, para lograr la transformación de su barrio y/o sector, por medio de actividades autogestionadas que responden a sus necesidades y aspiraciones; a través de la aplicación de una estrategia donde la organización y participación ciudadana son los pilares fundamentales de este accionar, con lo que se logra que la sociedad, tanto urbana como rural, comience a comprender la importancia de identificar los problemas que afectan y buscar soluciones, no solo en su entorno, sino a través de los organismos competentes.

Paralelamente y de acuerdo a lo expresado por gestores de los proyectos, se pudo evidenciar que la premisa de estos programas de RS, radica en brindar ayuda de forma desinteresada y voluntaria, sin limitaciones frente a los inconvenientes económicos que en la actualidad afrontan, sumado a la presión por parte de la leyes impuestas actualmente, que obligan al cumplimiento de programas sociales, lo que se alinea con la teoría sobre RS expuesta, la misma que busca el desarrollo de sus grupos de interés tomando en cuenta los aspectos económico, social y ambiental.

Las estrategias y programas implementados en Ecuador han sido desarrollados en función de las necesidades sociales de los ciudadanos y de las exigencias de las 
normativas y las leyes, lo que ha llevado a elaborar mecanismos para incrementar la transparencia y el control de la corrupción. De esta manera, la aplicación de las herramientas de evaluación en este análisis, indican que el concepto de responsabilidad social y la práctica de la transparencia y rendición de cuentas, están relacionados con informar sobre los aspectos económicos, más de carácter legal impositivo, y los aspectos medio ambientales, generalmente asociados a los impactos en el desarrollo territorial. Esta innovación pública mediante el recurso de estrategias de Responsabilidad Social contribuye al progreso de la sociedad mediante los valores de transparencia, ética política, participación ciudadana e igualdad, generando más confianza entre el Estado y la Sociedad. 


\section{Referencias}

Argandoña, A., \& Isea Silva, R. (2011). ISO 26000 una guía de responsabilidad de las organizaciones. Instituto Politécnico Nacional.

Asamblea Nacional. (18 de Mayo de 2004). Ley Orgánica de Transparencia y Acceso a la información pública. Quito, Pichincha, Ecuador: Reg. Oficial No. 337.

Asamblea Nacional. (20 de Octubre de 2008). Constitución de la República del Ecuador. Quito, Ecuador.

Banco Mundial. (2000). Anticorruption in Transition: A Contribution to the Policy Debate. Washington, D.C.: World Bank-International Bank for Reconstruction and Development.

Canovas García, J., \& Mateos García, J. (2014). Responsabilidad Social Corporativa: más que un modelo de gestión, un reto para las Administraciones Públicas. Escuela de Formación e Innovación, 8.

CCE, C. d. (18 de Julio de 2001). Libro Verde. Fomentar un marco europeo para la responsabilidad social de las empresas. Obtenido de http://www.europarl.europa. eu/meetdocs/committees/deve/20020122/com(2001)366_es.pdf

CEPAL, C. E. (Abril de 2004). Responsabilidad Social corportiva en América Latina: una visión empresarial. Obtenido de https://repositorio.cepal.org/bitstream/ handle/11362/5621/1/S044214_es.pdf

Contraloría General, d. E. (14 de Diciembre de 2009). Normas de Control Interno de la Contraloría General del Estado. Quito, Pichincha, Ecuador: Registro Oficial No. 87 .

Fox, T., Ward, H., \& Howard, B. (2002). Public sector roles in strengthening corporate social responsability: a baseline study. Banco Mundial.

González, M., \& León, M. (2013). Responsabilidad Social Empresarial: Aproximación a las líneas de actuación públicas de la Unión Europea, América Latina y el Caribe. Actualidad Económica, 4-11.

GRI Empowering Sustainable Decisions. (s.f.). Obtenido de www.globalreporting.org/ standards/

Hernández Sampiere, R., Fernández Collado, C., \& Baptista Lucio, P. (2006). Metodología de la investgación. México: McGraw-Hill Interamericana.

International Organization for Standardization. (s.f.). Obtenido de https://www. iso.org/standards.html 
Lozano, J., Albareda, L., Ysa, Rosher, \& Marcuccio. (2005). Políticas Públicas más alla de la regulación y la voluntariedad. Los gobiernos y la responsabilidad social de las empresas.

Navarro Galera, A., Alcaraz Quilez, F., \& Ortiz, D. (2010). La divulgación de la información sobre responsabilidad corporativa en administraciones públicas: un estudio empírico en gobiernos locales. Revista de Contabilidad, 285-314.

Navarro Galera, A., Ruiz Lozano, M., De Los Rios Berjillos, A., \& Tirado Valencia, P. (2013). La responsabilidad social corporativa en los gobiernos locales: el caso de Reino Unido e Irlanda. Innovar.

Norma Técnica de Implementación y Operación de la metodología y herramienta de Gobierno por Resultados. (28 de Diciembre de 2011). Quito, Pichincha, Ecuador: Registro Oficial No. 606.

Observatorio de Responsabilidad Social Corporativa. (2003). Obtenido de : http://observatoriorsc.org/normas-sobre-las-responsabilidades-de-las-empresas-transnacionalesy-otras-empresas-comerciales-en-la-esfera-de-los-derechos-humanos/

OCDE. (2011). OECD.org. Obtenido de http://www.oecd.org/daf/inv/mne/oecdguidelinesformultinationalenterprises.htm

OIT. (2001). Oficina Internacional de Trabajo . Obtenido de http://www.ilo.org/wcmsp5/groups/public/---dgreports/---dcomm/documents/normativeinstrument/ kd00121es.pdf

ONU, O. d. (2015). Informe Bruntdland. Obtenido de www.un.org/sustainabledevelopment/es/

ONU, O. d. (s.f.). Organización de las Naciones Unidas. Obtenido de https://www. un.org/es/about-un/

Orjuela Cordova, S. (2011). La comunicación en la gestión de responsabilidad social empreasrial. Correspondencia y Análisis.

Puig i Campmany, M., \& Martínez i Hernández, A. (2008). La Responsabilidad Social de la Administración. Un reto para el siglo XXI. Desarrollo Económico, 49.

Ruiz, C., \& Ruiz, R. (2014). Responsabilidad Social Pública y Calidad Democrática. Derecho y Cambio Social, 4.

Senplades, S. N. (22 de Septiembre de 2017). Plan Nacional de Desarrollo 2017 2021. Toda una Vida. Quito, Pichincha, Ecuador.

United Nations Global Compact. (s.f.). Obtenido de www.unglobalcompact.org/whatis-gc/mission/principles 
Viteri, J., \& Jácome, M. (2011). La responsabilidad social como modelo de gestión empresarial. EIDOS, 3-9.

Zenck Huerta, M. C., Rios Rivera, I., Pogo García, L., \& Cueto Cedillo, C. (2017). Análisis de la transparencia sobre las políticas locales de responsabilidad social en Ecuador: Estudio de los casos de Quito, Guayaquil y Machala. Revista Iberoamericana de Contabilidad de Gestión. 
Universidad Técnica de Machala

Ediciones UTMACH

Primera edición en español 2020

PDF interactivo 



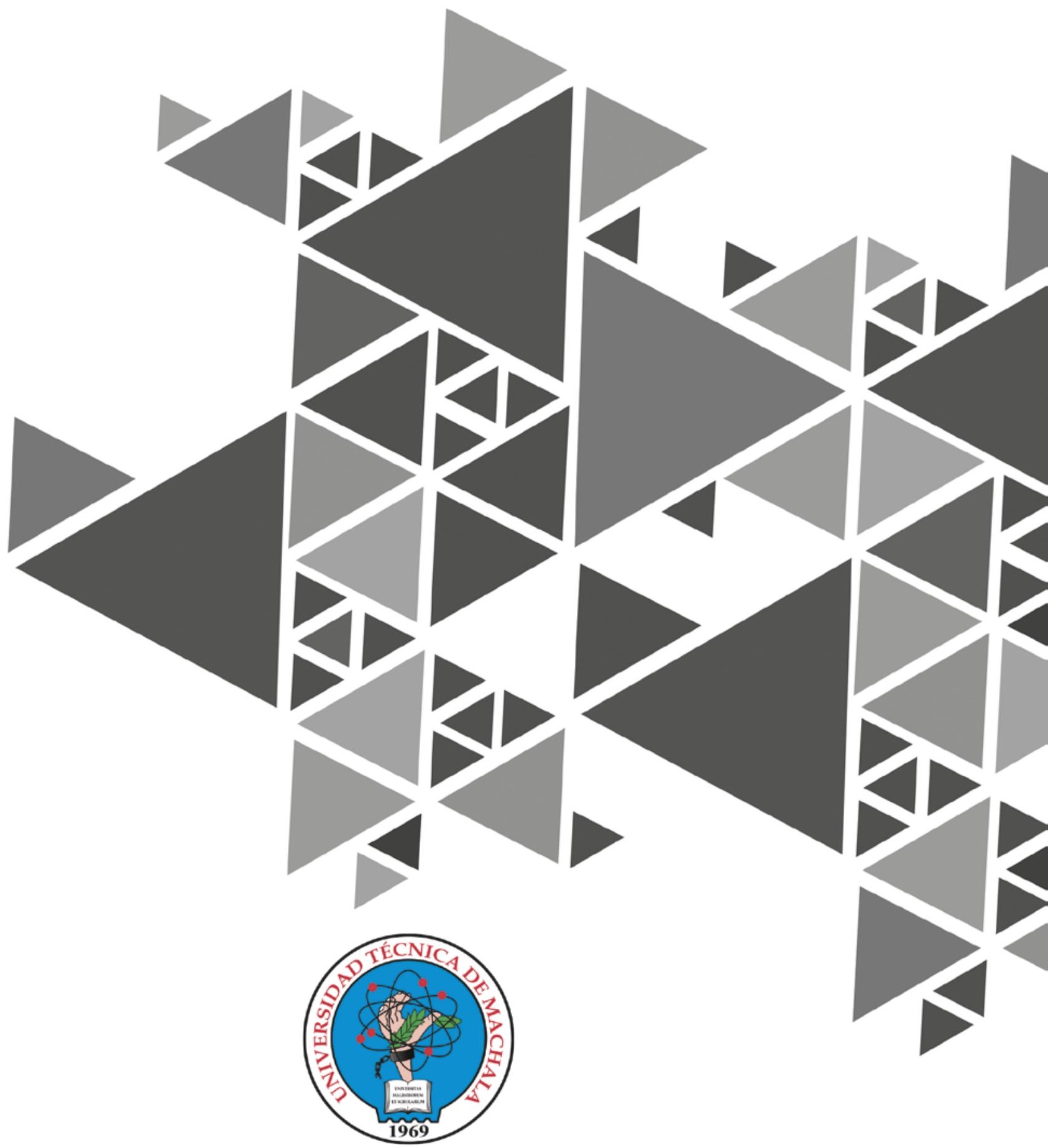

Universidad Técnica de Machala

Dirección de Investigación 